\title{
Gamma-ray line from Nambu-Goldstone dark matter in a scale invariant extension of the Standard Model
}

\author{
Jisuke Kubo, ${ }^{a}$ Kher Sham $\operatorname{Lim}^{b}$ and Manfred Lindner ${ }^{b}$ \\ ${ }^{a}$ Institute for Theoretical Physics, Kanazawa University, \\ Kanazawa 920-1192, Japan \\ ${ }^{b}$ Max-Planck-Institut für Kernphysik, \\ 69117 Heidelberg, Germany \\ E-mail: jik@hep.s.kanazawa-u.ac.jp, khersham.lim@mpi-hd.mpg.de, \\ lindner@mpi-hd.mpg.de
}

ABSTRACT: A recently proposed scale invariant extension of the standard model is modified such that it includes a Dark Matter candidate which can annihilate into gamma-rays. For that a non-zero $\mathrm{U}(1)_{Y}$ hypercharge $Q$ is assigned to the fermions in a QCD-like hidden sector. The Nambu-Goldstone bosons, that arise due to dynamical chiral symmetry breaking in the hidden sector, are cold Dark Matter candidates, and the extension allows them to annihilate into two photons, producing a $\gamma$-ray line spectrum. We find that the $\gamma$-ray line energy must be between $0.7 \mathrm{TeV}$ and $0.9 \mathrm{TeV}$ with the velocity-averaged annihilation cross section $10^{-30} \sim 10^{-26} \mathrm{~cm}^{3} / \mathrm{s}$ for $Q=1 / 3$. With a non-zero hypercharge $Q$, the hidden sector is no longer completely dark and can be directly probed by collider experiments.

Keywords: Beyond Standard Model, Phenomenological Models

ARXIV EPRINT: 1405.1052 


\section{Contents}

1 Introduction $\quad 1$

2 The model 3

3 Relic abundance of DM and its direct detection $\quad 6$

4 Restoring gauge invariance $\quad 9$

5 Monochromatic $\gamma$-ray line from DM annihilation $\quad 10$

6 Conclusion 12

$\begin{array}{ll}\text { A Least subtraction procedure } & 13\end{array}$

\section{Introduction}

With the discovery of the Higgs particle $[1,2]$ the standard model (SM) is now complete. However, the SM must be extended since it does not contain a Dark Matter (DM) candidate and since finite neutrino masses must also be included. From a pure theoretical point of view there exist also severe conceptual problems and one of them is that the SM cannot explain the origin of its energy scale. Theoretically, we can imagine a world without any energy scale, but in the real world of elementary particles scale invariance is broken. In the SM the mass term of the Higgs field is the only term in the Lagrangian that violates (at tree level) scale invariance. Although the SM does not explain the origin of its energy scale, the measured mass $m_{h}$ of the Higgs particle seems to suggest how to go beyond the SM, because this mass value together with the top quark mass implies that the SM remains perturbative at least up to the Planck scale [3-6]; an ultraviolet (UV) completion of the SM is not needed. Any extension which modifies the high energy behavior of the SM should therefore be well motivated (see e.g. [7]), since it may require a UV completion at lower scales.

Introducing an explicit Higgs mass term in the SM does not only break classical scale invariance, but it also leads to another severe issue known as the gauge hierarchy problem, namely the quadratic sensitivity of quantum corrections to high scales. It is therefore tempting to start from classically scale invariant theories where the SM scale emerges from dimensional transmutation. Various attempts to introduce an energy scale in this way exist in the literature [8]-[42]. Scale invariance is broken at the quantum level even in perturbation theory $[43,44]$, but it has been argued that the protective features of conformal symmetry are not completely destroyed [45]. Specifically logarithmic sensitivities would exist, while quadratic divergencies would be absent. 
We follow the idea that the energy scale in a classically scale invariant theory is generated by $\mathrm{D} \chi \mathrm{SB}$ in a QCD-like hidden sector, which is transmitted via a SM singlet messenger field to the SM sector [46-49]. ${ }^{1}$ So we assume that the fermions in the hidden sector are SM singlet and allow the presence of fundamental scalar fields. In fact, the messenger is assumed to be the simplest possibility, a real SM singlet scalar $S$. Note that this avoids the well known phenomenological problems of technicolor models and the model looks very much like the SM, since the hidden sector couples only via the Higgs portal.

The possibility that DM annihilates into $\gamma$-ray lines has recently received much attention and we want to discuss this possibility therefore in this paper. Specifically we consider a simple extension of the above mentioned model, where we assign a $\mathrm{U}(1)_{Y}$ hypercharge $Q$ to the hidden sector fermions such that they are electrically charged with a charge $Q$. Since the coupling is vector-like, no breaking of $\mathrm{U}(1)_{Y}$ is caused by $\mathrm{D} \chi \mathrm{SB}$ in the hidden sector. As we will see, this non-zero charge makes it possible that DM particles, which are the pseudo Nambu-Goldstone particles in this model, can be annihilated into two photons, producing a $\gamma$-ray line spectrum. Monochromatic $\gamma$-ray lines from DM annihilation exist in other DM models, too $[52,53]$, and in fact experimental searches for $\gamma$-ray lines have been undertaken with Fermi LAT [55, 56] and HESS [57] for a wide range of high energies. We find that the energy of the $\gamma$-ray line in our model lies between $0.7 \mathrm{TeV}$ and $0.9 \mathrm{TeV}$. (We are not aiming to explain the recent observations of the galactic $\mathrm{keV}$ X-ray $[58,59]$ here.) The upper limits on the velocity-averaged annihilation cross section $\langle v \sigma\rangle$ given by Fermi LAT and HESS constrain the electric charge $Q$ of the hidden fermions. We find that the $\langle v \sigma\rangle$ is $10^{-30} \sim 10^{-26} \mathrm{~cm}^{3} / \mathrm{s}$ for $Q=1 / 3$, which can well satisfy the experimental constraints of Fermi LAT and HESS. Since $\langle v \sigma\rangle$ is proportional to $Q^{4}$, our calculations can be simply extended to the case of an arbitrary $Q$.

In this model not only DM particles but also hidden baryons are stable. For an arbitrary $Q$ the electric charge of the hidden baryons are fractionally charged. Note that the consistent range of the $\mathrm{DM}$ mass between $0.7 \mathrm{TeV}$ and $0.9 \mathrm{TeV}$ is independent of $Q$ and hence the scale $\Lambda$ of the hidden sector is roughly fixed (regardless of $Q$ ), which means that the mass of the stable hidden baryons is $\sim 3 \mathrm{TeV}$. Using the fact that the hidden sector is basically described by a scaled-up QCD, we have found that the relic abundance of the hidden baryons $\Omega_{h B} h^{2}$ in the Universe is at most $10^{-4}$, which is independent of $Q$. This is sufficiently below the upper bound given in [60] and the constraint in the $Q$-DM mass plane given in [61] is also satisfied. Consequently there is practically no constraint (except for those from FermiLat and HESS) on the fractionally charged hidden baryons.

Since the hidden sector (strictly speaking it is no longer a hidden sector, because the fermions are electrically charged) can now communicate through gauge boson exchange (photon and $Z$ boson) with the SM sector, the hidden sector could be produced at the ILC. We postpone these interesting processes for future studies, as our main priority in this paper is to find a prescription to obtain gauge invariant amplitudes. This is because we approximate the strongly coupled QCD-like sector by the Nambu-Jona-Lasinio model (NJL) [6264] (see [65, 66] for reviews), which is defined with a finite cutoff $\Lambda$ that violates gauge

\footnotetext{
${ }^{1}$ See also $[50,51]$.
} 
invariance. To overcome this problem, we propose least subtraction procedure. In the NJL model the cutoff $\Lambda$ is a physical parameter and a finite $\Lambda$ is essential to describe effectively $\mathrm{D} \chi \mathrm{SB}$. We therefore stress that we keep the subtraction terms to the minimum necessary.

\section{The model}

We consider an extension of the model studied in [46-49] which consists of a hidden QCDlike sector coupled via a real singlet scalar $S$ to the SM. The fermion $\psi$ in the hidden sector belongs to the fundamental representation of the hidden gauge group $\mathrm{SU}(3)_{H}$. With this setting $\mathrm{D} \chi \mathrm{SB}$ in the hidden sector does not break the SM gauge symmetries, thereby avoiding the FCNC problem. This is one of the main differences to technicolor model. If we further assume that the Yukawa coupling $\bar{\psi} \psi S$ respects $\mathrm{SU}\left(N_{f}\right)_{V}$ flavor symmetry, there is only one coupling constant $y$ for the Yukawa coupling, so that in the hidden sector there are only two independent parameters; the gauge coupling constant $g_{H}$ and the Yukawa coupling $y$.

In extending the model we impose that neither the SM gauge symmetry nor the $\mathrm{SU}\left(N_{f}\right)_{V}$ flavor symmetry is broken in the hidden sector. If we further impose that the matter content remains unchanged, then there is a unique possibility for the extension that the hidden (Dirac) fermion carries a common $\mathrm{U}(1)_{Y}$ charge $Q .{ }^{2}$ This implies that the hidden sector Lagrangian of the extended model is written as

$$
\mathcal{L}_{\mathrm{H}}=-\frac{1}{2} \operatorname{Tr} F^{2}+\operatorname{Tr} \bar{\psi}\left(i \gamma^{\mu} \partial_{\mu}+g \gamma^{\mu} G_{\mu}+g^{\prime} Q \gamma^{\mu} B_{\mu}-y S\right) \psi,
$$

where $G_{\mu}$ is the gauge field for the hidden $\mathrm{QCD}$, and $B$ is the $\mathrm{U}(1)_{Y}$ gauge field. The trace is taken over the flavor as well as the color indices. The $\mathcal{L}_{\mathrm{SM}+S}$ part of the total Lagrangian $\mathcal{L}_{T}=\mathcal{L}_{\mathrm{H}}+\mathcal{L}_{\mathrm{SM}+S}$, which contains the SM gauge and Yukawa interactions along with the scalar potential

$$
V_{\mathrm{SM}+S}=\lambda_{H}\left(H^{\dagger} H\right)^{2}+\frac{1}{4} \lambda_{S} S^{4}-\frac{1}{2} \lambda_{H S} S^{2}\left(H^{\dagger} H\right),
$$

is unchanged. ${ }^{3} H^{T}=\left(H^{+},(h+i G) \sqrt{2}\right)$ is the SM Higgs doublet field, with $H^{+}$and $G$ as the would-be Nambu-Goldstone fields.

Here we follow [49] in which the NJL model is used to describe D $\chi$ SB in the hidden sector, restricting ourselves to $N_{c}=N_{f}=3$, because in this case the NJL model parameters, up-to an overall scale, can be fixed from hadron physics [66-69]. So at low energy we replace the Lagrangian $\mathcal{L}_{H}$ by

$$
\mathcal{L}_{\mathrm{NJL}}=\operatorname{Tr} \bar{\psi}\left(i \gamma^{\mu} \partial_{\mu}+g^{\prime} Q \gamma^{\mu} B_{\mu}-y S\right) \psi+2 G \operatorname{Tr} \Phi^{\dagger} \Phi+G_{D}(\operatorname{det} \Phi+\text { h.c. }),
$$

where

$$
B_{\mu}=\cos \theta_{W} A_{\mu}-\sin \theta_{W} Z_{\mu}, g^{\prime}=e / \cos \theta_{W}
$$

\footnotetext{
${ }^{2}$ The new gauge coupling contributes only to $\Pi_{Y Y}$ of the gauge boson self-energy diagrams so that the $S, T, U$ parameters remain unchanged.

${ }^{3}$ This classically scale invariant model is perturbatively renormalizable, and the Green's functions are infrared finite [70-72].
} 


$$
\Phi_{i j}=\bar{\psi}_{i}\left(1-\gamma_{5}\right) \psi_{j}=\frac{1}{2} \lambda_{j i}^{a} \operatorname{Tr} \bar{\psi} \lambda^{a}\left(1-\gamma_{5}\right) \psi,
$$

and $\lambda^{a}$ are the Gell-Mann matrices with $\lambda^{0}=\sqrt{2 / 3}$ 1. The last term in (2.3), which exhibits a six fermi interaction, is present due to chiral anomaly of the axial $\mathrm{U}(1)_{A}$. The chiral symmetry $\mathrm{U}(3)_{L} \times \mathrm{U}(3)_{R}$ is explicitly broken down to its diagonal subgroup $\mathrm{U}(3)_{V}=\mathrm{SU}(3)_{F} \times \mathrm{U}(1)_{V}$ by the Yukawa coupling with the singlet $S$. To deal with the non-renormalizable Lagrangian (2.3) we have used in [49] a self-consistent mean-field approximation which has been intensely studied by Hatsuda and Kunihiro [66-69] for hadron physics. The effective Lagrangian $\mathcal{L}_{\text {NJL }}$ has three dimensional parameters $G, G_{D}$ and the cutoff $\Lambda$, which have canonical dimensions of $-2,-5$ and 1 , respectively. Since the original Lagrangian $\mathcal{L}_{H}$ has only one independent scale, the parameters $G, G_{D}$ and $\Lambda$ are not independent. We obtain the NJL parameters for the hidden QCD from the upscaling of actual values of $G, G_{D}$ and the cutoff $\Lambda$ from QCD hadron physics. That is, we assume that the dimensionless combinations

$$
G^{1 / 2} \Lambda=2.0,\left(-G_{D}\right)^{1 / 5} \Lambda=2.1
$$

which are satisfied for hadrons, remain unchanged for a higher scale of $\Lambda$ [49].

In what follows we briefly outline the approximation method [66-69]. One assumes that the dynamics of the theory creates a chiral symmetry breaking condensate

$$
\left\langle 0\left|\bar{\psi}_{i} \psi_{j}\right| 0\right\rangle=-\frac{1}{4 G} \operatorname{diag}(\sigma, \sigma, \sigma),
$$

which is treated as a classical field $\sigma$. The vacuum $|0\rangle$ is defined by the annihilation operator of the constituent fermion $\psi$ in the background of the mean fields. We restrict our discussion (in a more complete treatment, one may add terms involving $\eta$ or $\rho$ mesons) to the mean fields collected in

$$
\varphi \equiv\left\langle 0\left|\bar{\psi}\left(1-\gamma_{5}\right) \lambda^{a} \psi\right| 0\right\rangle=-\frac{1}{4 G}\left(\operatorname{diag}(\sigma, \sigma, \sigma)+i\left(\lambda^{a}\right)^{T} \phi_{a}\right),
$$

where we denote the pseudo Nambu-Goldstone boson after spontaneous chiral symmetry breaking as $\phi_{a}$. These dark pions are stable due to flavor symmetry and they serve as good DM candidates. In the self-consistent mean field approximation one splits up the NJL Lagrangian (2.3) into the sum

$$
\mathcal{L}_{\mathrm{NJL}}=\mathcal{L}_{0}+\mathcal{L}_{I}
$$

where $\mathcal{L}_{I}$ is normal ordered (i.e. $\left\langle 0\left|\mathcal{L}_{I}\right| 0\right\rangle=0$ ), and $\mathcal{L}_{0}$ contains at most fermion bilinears which are not normal ordered. After some manipulations, one finds the following form for $\mathcal{L}_{0}[49]$ :

$$
\begin{aligned}
\mathcal{L}_{0}= & \operatorname{Tr} \bar{\psi} \gamma^{\mu}\left(i \partial_{\mu}+g^{\prime} Q B_{\mu}\right) \psi-\left(\sigma+y S-\frac{G_{D}}{8 G^{2}} \sigma^{2}\right) \operatorname{Tr} \bar{\psi} \psi-i \operatorname{Tr} \bar{\psi} \gamma_{5} \phi \psi-\frac{1}{4 G} \sum_{a=1}^{8} \phi_{a} \phi_{a} \\
& -\frac{3 \sigma^{2}}{8 G}+\frac{G_{D}}{8 G^{2}}\left(-\operatorname{Tr} \bar{\psi} \phi^{2} \psi+\sum_{a=1}^{8} \phi_{a} \phi_{a} \operatorname{Tr} \bar{\psi} \psi+i \sigma \operatorname{Tr} \bar{\psi} \gamma_{5} \phi \psi+\frac{\sigma^{3}}{2 G}+\frac{\sigma}{2 G} \sum_{a=1}^{8}\left(\phi_{a}\right)^{2}\right)
\end{aligned}
$$



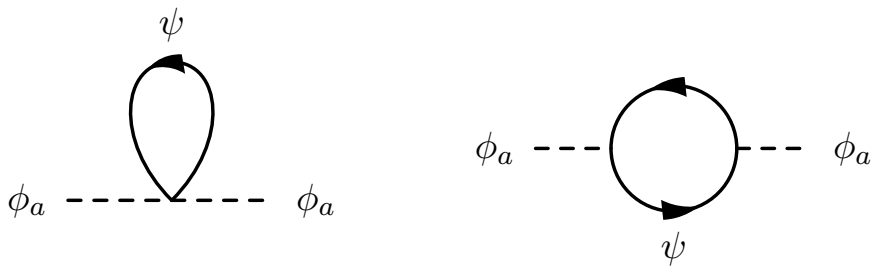

Figure 1. One-loop contributions of the heavy dark fermions to the DM mass.

Note that this Lagrangian no longer contains the four and six fermi interactions. At the non-trivial lowest order only $\mathcal{L}_{0}$ is relevant for the calculation of the effective potential, the DM mass $m_{\mathrm{DM}}$ and the DM interactions. The mass spectrum for all the CP-even particles, namely $h, S$ and $\sigma$ can be obtained from the minimum of the effective potential, once the free parameters of the model i.e. $y, \lambda_{H}, \lambda_{H S}, \lambda_{S}$ are given. See ref. [49] for more details in the calculation of the effective potential. The dimensionless couplings $y, \lambda_{H}, \lambda_{H S}, \lambda_{S}$ are required to satisfy perturbativity and vacuum stability up the Planck scale. Once the global minimum of the effective potential is obtained, the effective couplings between the bosons and the DM properties are determined. The $\mathrm{U}(1)_{Y}$ coupling does not contribute to the effective potential and the mass matrix for $h, S, \sigma$ in the lowest order.

As we have mentioned that the CP-odd pseudo Nambu-Goldstone bosons $\phi_{a}$ are the DM candidates for our model, let us investigate their properties in more details. Like the CP-even bosonized $\sigma$ field, the DM particles have no tree level kinetic term and their masses are defined as the zero of the inverse propagator

$$
\begin{aligned}
\Gamma_{\phi}\left(p^{2}\right)= & -\frac{1}{2 G}+\frac{G_{D}\langle\sigma\rangle}{8 G^{3}}+\frac{G_{D} N_{c}}{G^{2}} \int \frac{d^{4} k}{i(2 \pi)^{4}} \frac{M}{\left(k^{2}-M^{2}\right)} \\
& +2 N_{c}\left(1-\frac{G_{D}\langle\sigma\rangle}{8 G^{2}}\right)^{2} \int \frac{d^{4} k}{i(2 \pi)^{4}} \frac{\operatorname{Tr}(\not k-\not p+M) \gamma_{5}(\not k+M) \gamma_{5}}{\left((k-p)^{2}-M^{2}\right)\left(k^{2}-M^{2}\right)},
\end{aligned}
$$

where $M=\sigma+y S-G_{D} \sigma^{2} / 8 G^{2}$ is the constituent hidden sector fermion mass when all the CP-even scalar fields obtained their vacuum expectation values (VEV). The first two terms in eq. (2.10) stem from the tree level effective Lagrangian (2.9) while the heavy dark fermions contribute to the one-loop radiative correction for the DM inverse propagator. The relevant one-loop diagrams are given in figure 1. From eq. (2.10) the DM mass and its wave function renormalization constant can be calculated

$$
\Gamma_{\phi}\left(m_{\mathrm{DM}}^{2}\right)=0, Z_{\phi}^{-1}=\left.\frac{d \Gamma_{\phi}\left(p^{2}\right)}{d p^{2}}\right|_{p^{2}=m_{\mathrm{DM}}^{2}} .
$$

As $y \rightarrow 0$, the chiral symmetry of the fermions should be restored and $m_{\mathrm{DM}} \rightarrow 0,{ }^{4}$ hence the size of the DM mass is controlled by the Yukawa coupling $y$. The additional $\mathrm{U}(1)_{Y}$ coupling however does not contribute to the DM mass. 

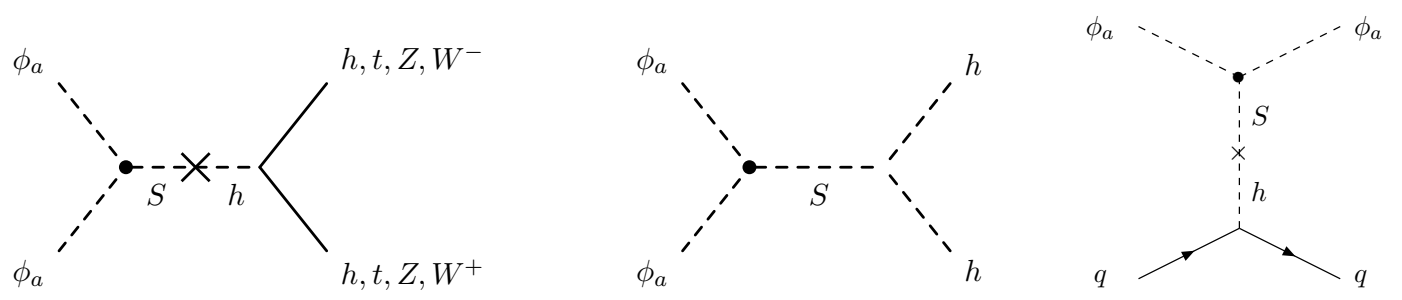

Figure 2. The left and middle diagrams are the s-channel DM annihilation diagrams. The right diagram contributes to the DM scattering off the nucleon. The coupling marked with a dot is a one-loop three-point vertex given in [49].

\section{Relic abundance of DM and its direct detection}

Before we start to compute the relic abundance $\Omega h^{2}$, let us discuss the parameter space. In our previous paper [49] the dimensionless coupling constants, $y, \lambda_{S, H S, H}$, are constrained by the vacuum stability and by the absence of the Landau pole. It turns out that with a non-zero $Q$ (at least for $Q \lesssim 1 / 3$ ) the allowed parameter space does not practically change. Note that the annihilation processes of DM occur at the one-loop level through the oneloop $\phi-\phi$ - $S$ amplitude and the one-loop $\phi-\phi-S$-S amplitude (if $m_{S}<m_{\mathrm{DM}}$ ). The $\phi-\phi-S$ amplitude can be calculated from the one-loop diagram

$$
\begin{aligned}
\Gamma_{\phi \phi S}= & 4 N_{c} y\left(1-\frac{G_{D}\langle\sigma\rangle}{8 G^{2}}\right)^{2} \int \frac{d^{4} k}{i(2 \pi)^{4}} \frac{\left.\operatorname{Tr}(\not k+M) \gamma_{5}(\not k-\not p+M)(\not k+\not)^{\prime \prime}+M\right) \gamma_{5}}{\left(\left(k-M^{2}\right)\left(k^{2}-M^{2}\right)\left(\left(k+p^{\prime}\right)^{2}-M^{2}\right)\right.} \\
& +N_{c} y \frac{G_{D}}{4 G^{2}} \int \frac{d^{4} k}{i(2 \pi)^{4}} \frac{\operatorname{Tr}\left(\not k-\not p^{\prime}+M\right)(\not k+\not p+M)}{\left(\left(k-p^{\prime}\right)^{2}-M^{2}\right)\left((k+p)^{2}-M^{2}\right)},
\end{aligned}
$$

which is crucial for determining the relic abundance and the direct detection cross section of the DM. The momenta $p, p^{\prime}$ represent the incoming momenta of the dark pions. The one-loop effective couplings are represented as $\bullet$ in figure 2 . These amplitudes are small for small $y$ as the amplitudes scale like $\mathcal{A}(\phi \phi \rightarrow S) \sim y$ and $\mathcal{A}(\phi \phi \rightarrow S S) \sim y^{2}$ respectively. As mentioned above, the size of the DM mass is controlled by $y$, i.e. a small $y$ implies a small DM mass and for a larger $y \gtrsim 0.2$ the DM mass $m_{\mathrm{DM}}$ can become larger than the fermion constituent mass $M$, which will develop imaginary parts in these one-loop amplitudes. In [49] we forbad the occurrence of the imaginary parts, yielding an upper bound on $y$ for a given set of $\lambda_{S, H S, S}$. As the parameter $y$ is bounded from above, the $\phi-\phi-S$-S amplitude contributes negligibly to the relic abundance calculation and only the $\phi-\phi-S$ amplitudes are important. However in this parameter space we have found that the only way to enhance the annihilation rate of DM is via a resonance effect in the s-channel annihilation processes shown in figure 2 (left and middle). That is, $2 m_{\mathrm{DM}} \simeq m_{S}$ has to be satisfied. The direct detection rate of DM is however strongly suppressed $\left(\lesssim 10^{-48} \mathrm{~cm}^{2}\right)$ because it is a t-channel process shown in figure 2 (right), constraining the parameter space into phenomenologically unattractive corner.

\footnotetext{
${ }^{4}$ In this case $\phi$ is a true Nambu-Goldstone boson.
} 

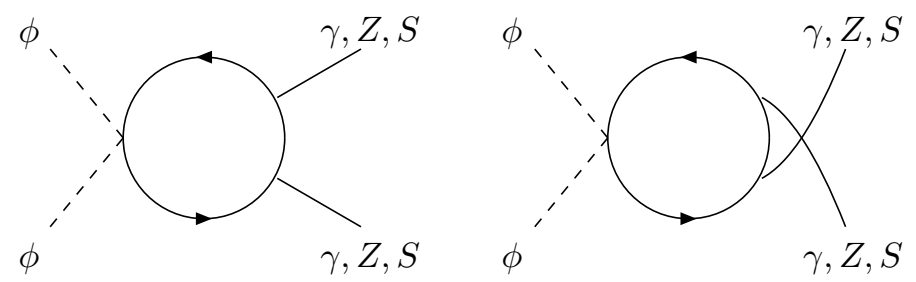

Figure 3. The DM annihilation with $\phi \phi \bar{\psi} \psi$ coupling (a).

In this paper we allow the occurrence of the imaginary parts in the one-loop diagrams, as they are related to the real parts due to the dispersion relation, which has proven to be successful in describing the QCD hadron physics (see [66] for instance). We set the upper bound at $m_{\mathrm{DM}}<2 M$, which should be compared with $m_{\eta^{\prime}}=0.958 \mathrm{GeV}$ and $M_{s}=0.5 \mathrm{GeV}$ in the usual QCD physics, where $M_{s}$ is the constituent mass of the strange quark. In fact, in the optimistic range $y \gtrsim 0.4$ with $m_{S}<m_{\mathrm{DM}}$, the $\phi-\phi-S-S$ amplitude (which is generated at one-loop as shown in figure $3 \sim 5$ ) is no longer small and can become large enough to give a correct relic abundance of DM. Therefore, we choose below the parameter space $y \gtrsim 0.4$ and open the channel $\phi \phi \rightarrow S S$. In this parameter region, the s-channel processes contributed by $\phi-\phi-S$ amplitudes are negligibly suppressed and can be ignored. The annihilation diagrams in figure $3 \sim 5$ for $\phi \phi \rightarrow S S$ yield

$$
\mathcal{A}(\phi \phi \rightarrow S S)=2 N_{c} y^{2}\left[\frac{G_{D}}{4 G^{2}} I_{a}+\left(1-\frac{G_{D}\langle\sigma\rangle}{8 G^{2}}\right)^{2}\left(4 I_{b}+2 I_{c}\right)\right],
$$

where $I_{i}$ represents the integral for the respective $i$ th loop diagram in figure $3 \sim 5$. We obtain the DM annihilation cross section

$$
\langle v \sigma(\phi \phi \rightarrow S S)\rangle=\frac{Z_{\phi}^{2}}{32 \pi m_{\mathrm{DM}}^{3}}|\mathcal{A}(\phi \phi \rightarrow S S)|^{2}\left(1-\frac{m_{S}^{2}}{m_{\mathrm{DM}}^{2}}\right)^{1 / 2},
$$

where $Z_{\phi}$ is given in eq. (2.11). We do not include the annihilation modes into $\gamma \gamma, \gamma Z, Z Z$ as the annihilation cross section of these modes is proportional to $\alpha^{2} Q^{4}$ (see eq. (5.5)) while the DM annihilation cross section to $S$ particles is dominated by $y^{4}$. Unless the electric charge $Q \gtrsim 1$, the annihilation modes into $\gamma \gamma, \gamma Z, Z Z$ can be ignored in the relic abundance calculation (see also the comment in the footnote on page 12). The annihilations into these modes are calculated in the next section. We find, imposing the constraint on the relic abundance $\Omega h^{2}=0.1187 \pm 0.005(3 \sigma)$ [73], that the spin independent annihilation cross section is just below the XENON100 [74] and LUX [75] constraints and above the XENON1T sensitivity [76]. This is shown in figure 6 .

The DM mass $m_{\mathrm{DM}}$ is constrained in the present model. The lower limit $m_{\mathrm{DM}} \gtrsim$ $0.7 \mathrm{TeV}$ comes from the fact that $y$ has to be large enough so that the size of the annihilation process $\phi \phi \rightarrow S S$ yields a correct relic abundance of DM. If on the other hand $y$ is too large, the annihilation cross section into two singlet scalars becomes too large so that the 

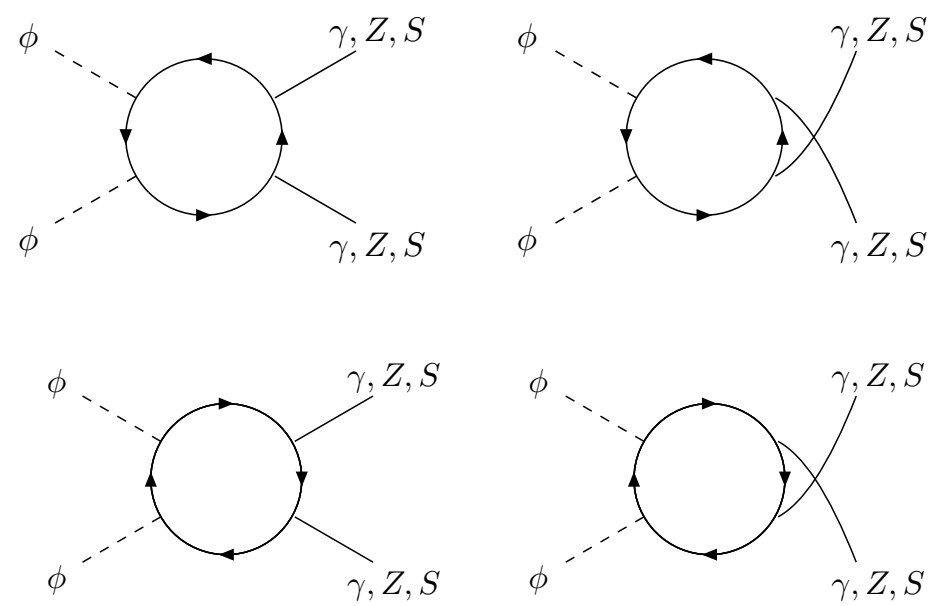

Figure 4. The DM annihilation (b).
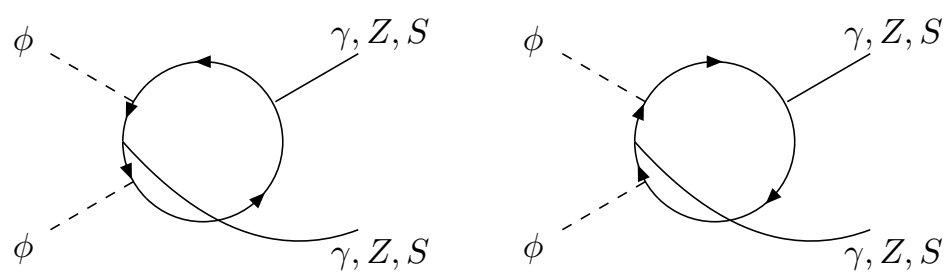

Figure 5. The DM annihilation (c).

relic abundance falls below the observed value. The Yukawa coupling $y$ is also constrained from above to avoid the triviality bound, which gives the upper limit $m_{\mathrm{DM}} \lesssim 0.9 \mathrm{TeV}$.

Note that because of the $\mathrm{SU}(3)_{V}$ flavor symmetry and an accidental $\mathrm{U}(1)_{\mathrm{hB}}$ (hidden baryon number), not only the DM candidates, but also the lightest hidden baryons are stable. In the case that $N_{c}=N_{f}=3$ in the hidden sector and $Q=1 / 3$ for the hidden fermions, there is no stable hidden hadron with a fractional electric charge. The hidden mesons for our model are neutral, while the charge of the hidden baryons formed by three hidden fermions is one if $Q=1 / 3$. There might be a tiny amount of relic stable hidden baryons and anti-baryons in the universe, which if a large number of them are not annihilated, could spoil the large scale structure formation. Let us roughly estimate fraction of this hidden baryon. As the hidden sector is described by a scaled-up QCD, so that because the coupling $G_{\phi B \bar{B}}$ is dimensionless, the hidden meson-baryon coupling $G_{\phi B \bar{B}}$ is approximately the same as in QCD, i.e. $G_{\phi B \bar{B}} \sim 13$. Using this fact, we have estimated the relic abundance $\Omega_{h B} h^{2}$ of the hidden baryons to be $\sim 10^{-4}$ for the hidden baryon mass of $3 \mathrm{TeV}$. Note that this result is independent of $\mathrm{Q}$. Therefore, we may fairly ignore the stable charged hidden baryons in discussing the relic abundance of DM. 

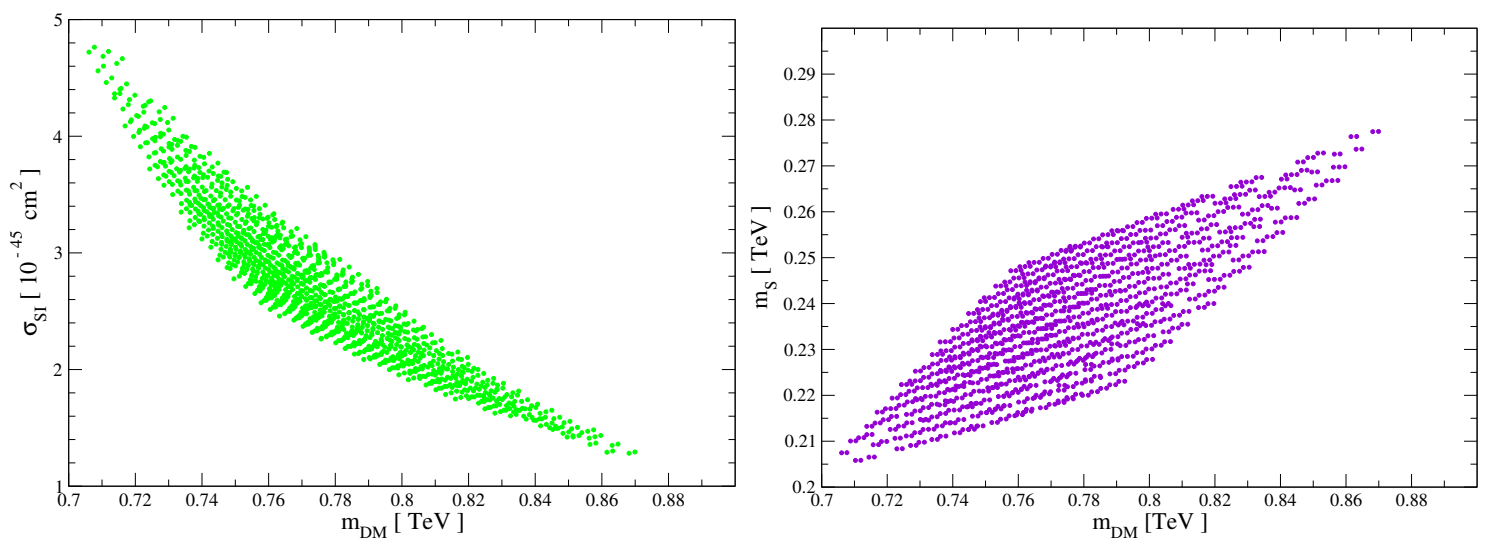

Figure 6. Left: the spin-independent cross section off nucleon against $m_{\mathrm{DM}}$, where $\Omega h^{2}=$ $0.1187 \pm 0.005(3 \sigma)[73]$ is imposed. The XENON100 [74] and LUX [76] limits are $\sim 10^{-44} \mathrm{~cm}^{2}$ for $m_{\mathrm{DM}}=0.7 \mathrm{TeV}$, while the XENON1T sensitivity is two orders of magnitudes higher than that of XENON100 [76]. Right: the mass of the singlet $S$ against $m_{\mathrm{DM}}$. If $m_{\mathrm{DM}}<m_{S}$, then $\sigma_{\mathrm{SI}} \lesssim 10^{-48} \mathrm{~cm}^{2}[49]$.

\section{Restoring gauge invariance}

The cutoff $\Lambda$ breaks gauge invariance explicitly and to restore gauge invariance we have to subtract non-gauge invariant terms from the original amplitude. In renormalizable theories there is no problem to define a finite renormalized gauge invariant amplitude, i.e. In the limit of $\Lambda \rightarrow \infty$ the gauge non-invariant terms are a finite number of local terms, which can be cancelled by the corresponding local counter terms so that the subtracted amplitude is, up to its normalization, independent of the regularization scheme (see for instance [77]). To achieve such a uniqueness in cutoff theories, one needs an additional prescription. For instance, we can define the real part of an amplitude using dispersion relation, as it was done in the original paper by Nambu and Jona-Lasinio [63, 64] (see also [66]). This procedure yields a gauge invariant real part of the amplitude in one-loop, because the imaginary part of the amplitude is gauge invariant in one-loop order. In this method, however, the one-loop tadpole diagram cannot be reproduced from its imaginary part as the tadpole diagram does not contain any imaginary part. Another way ${ }^{5}$ is to utilize a gauge invariant regularization such as the Pauli-Villars regularization [65, 79] which preserves gauge invariance by construction but breaks chiral symmetry explicitly. The drawback is however, for a finite regulator mass, it is not clear whether the breaking of chiral symmetry results from the regulator or from non-perturbative effect. Moreover, the regulator fields are "ghost" fields, which are not completely decoupled at a finite cutoff $\Lambda$.

We will propose another method, which we call "least subtraction procedure". In the NJL model as a cutoff theory the cutoff $\Lambda$ is a physical parameter, and a finite $\Lambda$ is essential to describe effectively $\mathrm{D} \chi \mathrm{SB}$. If we subtract too much from the amplitude to restore gauge invariance, we may lose information on non-perturbative effects. Therefore, we stress that

\footnotetext{
${ }^{5} \zeta$-function regularization was also used in [78] to obtain a gauge invariant effective potential in the presence of the electromagnetic field as an external field.
} 
we keep the subtraction terms to the minimum as necessary. The details of least subtraction procedure is given in appendix A, where we consider the photon self-energy, the $S-\gamma-\gamma$ as well as the $\phi-\phi-\gamma-\gamma$ vertex functions. The results are applied to the next section for the calculation of the DM annihilation cross section into two $\gamma$ 's.

\section{Monochromatic $\gamma$-ray line from DM annihilation}

The charge $Q$ of the hidden fermion is a free parameter. It can be constrained from the indirect detection of DM, e.g. the upper bound on $\sigma(\phi \phi \rightarrow \gamma \gamma)$ for $\gamma$-ray lines given in [5557]. The four-point $\phi-\phi-\gamma-\gamma$ coupling $^{6}$ is generated at one-loop as is shown in figure $3 \sim 5$, which predicts the DM annihilation into two monochromatic photons of energy $m_{\mathrm{DM}}$. Similar processes have been calculated in a universal extra dimension model [53], for instance. In appendix $A$ it is shown how to restore gauge invariance of the four-point amplitude, with the result given in (A.32). If we neglect the mass of $Z$ against $m_{\mathrm{DM}}$, the four-point functions $\mathcal{A}_{\mu \nu}^{R}(\gamma Z)$ and $\mathcal{A}_{\mu \nu}^{R}(Z Z)$ can be approximated by (A.32) as well, with the replacement of $e^{2}$ by $-e^{2} t_{W}$ and $e^{2} t_{W}^{2}$, respectively.

For $p=p^{\prime}=\left(m_{\mathrm{DM}}, \mathbf{0}\right)$ the photon momenta take the form $k=\left(m_{\mathrm{DM}}, \mathbf{k}\right)$ and $k^{\prime}=$ $\left(m_{\mathrm{DM}},-\mathbf{k}\right)$, with their polarization tensors $\epsilon(k)=(0, \boldsymbol{\epsilon}(k))$ and $\epsilon\left(k^{\prime}\right)=\left(0, \boldsymbol{\epsilon}\left(k^{\prime}\right)\right)$ satisfying

$$
\begin{aligned}
& 0=\epsilon(k) \cdot k=\epsilon(k) \cdot k^{\prime}=\epsilon(k) \cdot p=\epsilon(k) \cdot p^{\prime} \\
& 0=\epsilon\left(k^{\prime}\right) \cdot k=\epsilon\left(k^{\prime}\right) \cdot k^{\prime}=\epsilon\left(k^{\prime}\right) \cdot p^{\prime}=\epsilon\left(k^{\prime}\right) \cdot p^{\prime},
\end{aligned}
$$

respectively. Therefore, only $g_{\mu \nu}$ terms of the subtracted gauge invariant four-point function $\Gamma_{\mu \nu}(\gamma \gamma)$ contributes:

$$
\Gamma_{\mu \nu}(\gamma \gamma)=g_{\mu \nu}\left(\mathcal{A}^{R(a)}+\mathcal{A}_{g}^{R}\right)=i \frac{\alpha}{\pi} Q^{2} g_{\mu \nu} \mathcal{A}(\gamma \gamma),
$$

where $\mathcal{A}^{R(a)}$ (defined in (A.12)) is the contribution from figure 3 , while $\mathcal{A}_{g}^{R}$ (defined in (A.28)) is the contribution from figure 4 and 5 . The other ones can be approximated as

$$
\Gamma_{\mu \nu}(a b) \simeq i \frac{\alpha}{\pi} Q^{2} g_{\mu \nu} \mathcal{A}(\gamma \gamma) \times\left\{\begin{array}{cc}
a & b \\
-t_{W} & \gamma Z \\
t_{W}^{2} & Z Z
\end{array} .\right.
$$

Then (the s-wave part of) the corresponding velocity-averaged annihilation cross sections are given by

$$
\langle v \sigma(\phi \phi \rightarrow a b)\rangle=\frac{\alpha^{2} Q^{4} Z_{\phi}^{2}}{16 \pi^{3} m_{\mathrm{DM}}^{2}} \mathcal{A}^{2}(\gamma \gamma) \times\left\{\begin{array}{cc} 
& a b \\
& \gamma \gamma \\
t_{W}^{2}\left(1-m_{Z}^{2} / 4 m_{\mathrm{DM}}^{2}\right) & \gamma Z \\
(3 / 4) t_{W}^{4}\left(1-m_{Z}^{2} / m_{\mathrm{DM}}^{2}\right)^{1 / 2} & Z Z
\end{array}\right.
$$

where $Z_{\phi}$ is the wave function renormalization constant which is given in [49]. The energy $E_{\gamma}$ of $\gamma$-ray line produced in the annihilation into $\gamma Z$ is $m_{\mathrm{DM}}\left(1-m_{Z}^{2} / 4 m_{\mathrm{DM}}^{2}\right)$. In practice,

\footnotetext{
${ }^{6}$ The $\mathrm{U}(1)_{Y}$ gauge invariance and the $\mathrm{SU}(3)_{F}$ flavor symmetry together with the reality of $\phi$ forbid the existence of the $\phi \phi B_{\mu}$ coupling.
} 

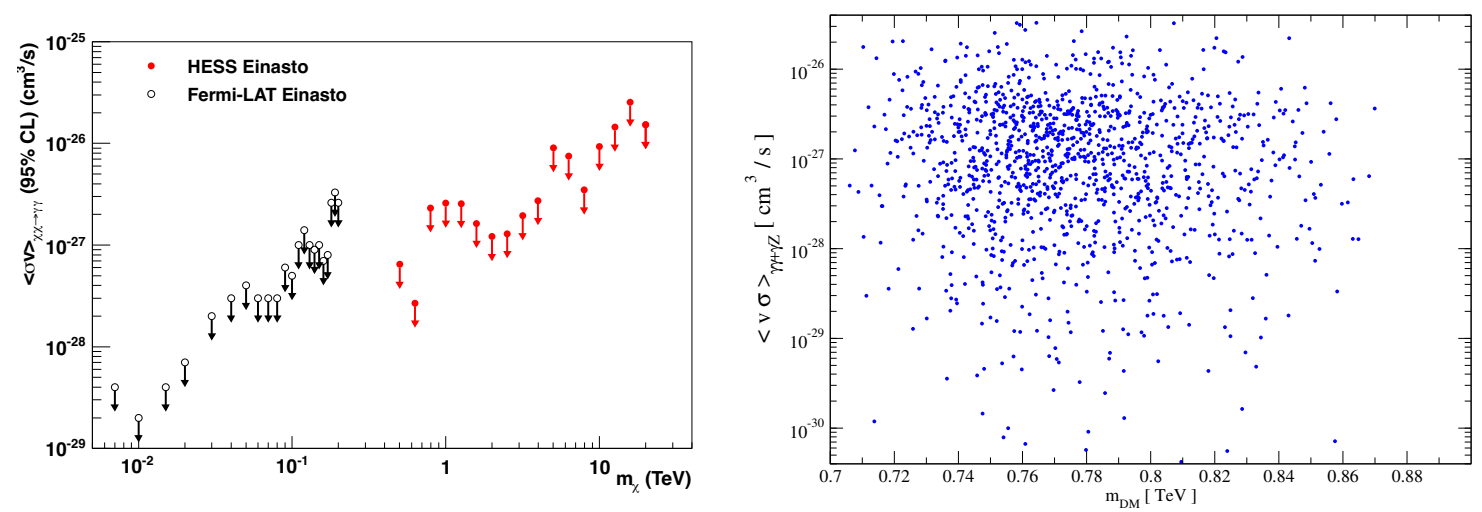

Figure 7. Left: the Fermi Lat [56] (black) and HESS [55] (red) upper bounds on the velocityaveraged DM annihilation cross section for monochromatic $\gamma$-ray lines, where this graph is taken from [55]. Right: the velocity-averaged DM annihilation cross section $\langle v \sigma\rangle_{\gamma \gamma+\gamma Z}$ as a function of $m_{\mathrm{DM}}$ with $Q=1 / 3$, where $\Omega h^{2}=0.1187 \pm 0.005(3 \sigma)$ [73] is imposed. Since $\langle v \sigma\rangle_{\gamma \gamma+\gamma Z}$ is proportional to $Q^{4}$, our calculations can be simply extended to the case of an arbitrary $Q$.

however, due to finite detector energy resolution this line cannot be distinguished from the $E_{\gamma}=m_{\mathrm{DM}}$ line. Therefore, we simply add both cross sections. So we compute $\langle v \sigma\rangle_{\gamma \gamma+\gamma Z}=\langle v \sigma(\phi \phi \rightarrow \gamma \gamma)\rangle+\langle v \sigma(\phi \phi \rightarrow \gamma Z)\rangle$ with $Q=1 / 3$ as a function of $m_{\mathrm{DM}}$ for different values of $\lambda_{H}, \lambda_{S}$ and $\lambda_{H S}$. As noticed in the previous section, we have not included the annihilation modes into $\gamma \gamma, \gamma Z, Z Z$ in calculating the relic abundance. In this way we can obtain a separate information on the size of the annihilation cross section producing the line $\gamma$-ray spectrum of DM in this model. ${ }^{7}$

As we see from figure 7 (left) strong constraints are given for $m_{\mathrm{DM}} \simeq 0.6(0.5) \mathrm{TeV}$ : $\langle v \sigma\rangle_{\gamma \gamma+\gamma Z} \lesssim 3(7) \times 10^{-28} \mathrm{~cm}^{3} / \mathrm{s}$. Since our DM is heavier than $0.7 \mathrm{TeV}$ (see figure 6), these strong constraints do not apply. Above $0.7 \mathrm{TeV}$, the upper bound is about one order of magnitude larger than that for $m_{\mathrm{DM}}=0.6 \mathrm{TeV}$, so that the constraints can well be satisfied even for $Q>1 / 3$, as we can see from figure 7 (right). An interesting feature of the present model is that the $\gamma$-ray line energy is constrained between $\sim 0.7 \mathrm{TeV}$ and $\sim 0.9 \mathrm{TeV}$, because the $\mathrm{DM}$ mass $m_{\mathrm{DM}}$ is constrained as it is explained in the previous section. Another feature of the model related to $\gamma$-ray lines is that the production cross section of $\gamma$-ray lines is in the same order in $1 / N$ expansion (i.e. in one-loop order) as the total annihilation cross section of DM. That is, $\langle v \sigma\rangle_{H H, f \bar{f}, W W, \ldots} \sim\langle v \sigma\rangle_{\gamma \gamma+\gamma Z}$ in the present model. This is similar to one of three exceptions, forbidden channels, considered in [80]. In the case of the forbidden channels the tree-level processes are kinematically forbidden, which should be contrasted to the present case in which the Nambu-Goldstone DM has no contact with the messenger field $S$ at the tree-level.

\footnotetext{
${ }^{7}$ The contribution can become important for $\langle v \sigma\rangle_{\gamma \gamma+\gamma Z} \gtrsim\langle v \sigma(\phi \phi \rightarrow S S)\rangle \simeq 8 \times 10^{-27} \mathrm{~cm}^{3} / \mathrm{s}$. But this approximate inequality can not be satisfied for $m_{\mathrm{DM}}$ between $0.7 \mathrm{TeV}$ and $0.9 \mathrm{TeV}$, if the HESS constraint for $m_{\mathrm{DM}}=0.8 \mathrm{TeV}$, i.e. $\langle v \sigma\rangle_{\gamma \gamma+\gamma Z} \lesssim 2 \times 10^{-27} \mathrm{~cm}^{3} / \mathrm{s}$, is satisfied for this range of $m_{\mathrm{DM}}$. If the HESS constraint for $m_{\mathrm{DM}}=0.8 \mathrm{TeV}$ does not apply and there is no cosmological constraint for this range of $m_{\mathrm{DM}}$, we should control the size of $\langle v \sigma\rangle_{\gamma \gamma+\gamma Z}$ by varying $Q$ when the approximate inequality above is satisfied.
} 
The differential $\gamma$-ray flux is given by

$$
\frac{d \Phi}{d E_{\gamma}} \propto\langle v \sigma\rangle_{\gamma \gamma} \frac{d N^{\gamma \gamma}}{d E_{\gamma}}+\langle v \sigma\rangle_{\gamma Z} \frac{d N^{\gamma z}}{d E_{\gamma Z}} \simeq\langle v \sigma\rangle_{\gamma \gamma+\gamma Z} \delta\left(E_{\gamma}-m_{\mathrm{DM}}\right)
$$

Prospects observing such line spectrum is discussed in detail in [53, 54]. Obviously, with an increasing energy resolution the chance for the observation increases. Observations of $\gamma$-ray lines of energies between $\sim 0.7 \mathrm{TeV}$ and $\sim 0.9 \mathrm{TeV} \mathrm{TeV}$ not only fix the charge of the hidden sector fermion, but also yields a first experimental hint on the hidden sector.

\section{Conclusion}

The Nambu-Goldstone theorem predicts in the presented model for the hidden sector, where chiral symmetry is dynamically broken and hence a scale is created, the existence of a DM candidate. This generated scale is transmitted to the SM sector via a real SM singlet scalar $S$ to trigger spontaneous breaking of electroweak gauge symmetry. With a non-zero $\mathrm{U}(1)_{Y}$ hypercharge $Q$ of the hidden sector fermion the hidden sector is no longer dark, and new possibilities to test experimentally the hidden sector are open. We studied in this paper the possibility of DM annihilation and found that this model allows DM to annihilate into two photons, producing a $\gamma$-ray line spectrum. We found that the $\gamma$-ray line energy must be between $0.7 \mathrm{TeV}$ and $0.9 \mathrm{TeV}$ with the velocity-averaged annihilation cross section $10^{-30} \sim 10^{-26} \mathrm{~cm}^{3} / \mathrm{s}$ for $Q=1 / 3$, which satisfies easily the recent limits given by Fermi LAT $[55,56]$ and HESS [57].

With a non-zero $Q$ the hidden sector is doubly connected with the SM sector. The connection via photon and $Z$ opens possibilities to probe the hidden sector at collider experiments such as $e^{+} e^{-}$collision [81]. In the parameter range, where the annihilation of DM into two singlets $S S$ is dominant and a correct relic abundance of DM is obtained, the constituent mass $M$ of the fermion is comparable with $m_{\mathrm{DM}}$, i.e. $0.7 \mathrm{TeV} \lesssim M \lesssim 0.9 \mathrm{TeV}$. This is the energy region of hidden hadron physics and the scale of the hidden sector itself is $\sim 0.7 \mathrm{TeV}$, compared to $\Lambda_{\mathrm{QCD}} \approx 1 \mathrm{GeV}$. The hidden strong interaction becomes therefore perturbative at about one order of magnitude above this energy region, $\gtrsim 10 \mathrm{TeV}$, and the hidden fermion becomes massless and could be produced directly to yield hidden sector jets at collider experiments.

\section{Acknowledgments}

We would like to thank Teiji Kunihiro for useful discussions. J. K. would like to thank the theory group of the Max-Planck-Institut für Kernphysik in Heidelberg for their hospitality. J. K. is partially supported by the Grant-in-Aid for Scientific Research (C) from the Japan Society for Promotion of Science (Grant No.22540271). K. S. L. acknowledges support by the International Max Planck Research School for Precision Tests of Fundamental Symmetries. 


\section{A Least subtraction procedure}

Here we elucidate least subtraction procedure which can be applied to any cutoff theory in principle to obtain gauge invariant amplitudes. The basic idea is to keep the subtraction terms to the minimum necessary. This works as follows. Consider an unsubtracted amplitude

$$
\mathcal{A}_{\mu_{1} \ldots \mu_{n_{g}}}\left(\Lambda ; k_{1} \ldots k_{n_{g}}, p_{1} \ldots p_{n_{s}}\right),
$$

with $n_{g}$ photons and $n_{s}$ scalars (scalars and axial scalars). ${ }^{8}$ Expand the amplitude in the external momenta $k$ 's and $p$ 's:

$$
\mathcal{A}_{\mu_{1} \ldots \mu_{n_{g}}}=\sum_{m=0} \mathcal{A}_{\mu_{1} \ldots \mu_{n_{g}}}^{(m)}
$$

where $\mathcal{A}_{\mu_{1} \ldots \mu_{n_{g}}}^{(m)}$ consists of $m$-th order monomials of the external momenta. In general, $\mathcal{A}_{\mu_{1} \ldots \mu_{n g}}^{(0)}=\mathcal{A}_{\mu_{1} \ldots \mu_{n g}}(\Lambda ; 0, \cdots, 0)$ is non-vanishing and we can subtract it because it is not gauge invariant. We keep the tensor structure of $\mathcal{A}_{\mu_{1} \ldots \mu_{n g}}^{(0)}$ as the tensor structure of the counter terms for $\mathcal{A}_{\mu_{1} \ldots \mu_{n}}^{(m)}(m>0)$ until a new tensor structure for the counter terms is required. We continue this until no more new tensor structure is needed. At each step we stress the minimal number of the new tensor structures for the counter terms.

- Photon self-energy. As an example we consider the one-loop photon self-energy. Using the usual technique, introducing a Feynman parameter $x$ for the denominator of the propagators, going to the Euclidean momentum space, shifting the internal momentum appropriately, we obtain the unsubtracted self-energy tensor

$$
\begin{aligned}
\Pi_{\mu \nu}(\Lambda ; k)=i \frac{e^{2} Q^{2} N_{c} N_{f}}{8 \pi^{2}} \int_{0}^{1} d x & {\left[\frac{4 \Lambda^{2}(1-x) x\left(g_{\mu \nu} k^{2}-k_{\mu} k_{\nu}\right)-\Lambda^{4} g_{\mu \nu}}{\Lambda^{2}+B^{2}}\right.} \\
& \left.\left.-4\left(g_{\mu \nu} k^{2}-k_{\mu} k_{\nu}\right)(1-x) x \ln \left(1+\Lambda^{2} / B^{2}\right)\right)\right]
\end{aligned}
$$

with $B^{2}=M^{2}-(1-x) x k^{2}$. According to least subtraction procedure, we expand $\Pi_{\mu \nu}(\Lambda ; k)$ in $k$ and find

$$
\begin{aligned}
\Pi_{\mu \nu}(\Lambda ; k)= & i \frac{e^{2} Q^{2} N_{c} N_{f}}{8 \pi^{2}}\left[g_{\mu \nu} \mathcal{A}_{g}\left(\Lambda ; k^{2}\right)+k_{\mu} k_{\nu} \mathcal{A}_{k k}\left(\Lambda ; k^{2}\right)\right] \\
\mathcal{G}\left(\Lambda ; k^{2}\right)= & \mathcal{A}_{g}\left(\Lambda ; k^{2}\right)+k^{2} \mathcal{A}_{k k}\left(\Lambda ; k^{2}\right)=-\frac{\Lambda^{4}}{\Lambda^{2}+M^{2}}-\frac{k^{2} \Lambda^{4}}{6\left(\Lambda^{2}+M^{2}\right)^{2}}-\frac{k^{4} \Lambda^{4}}{30\left(\Lambda^{2}+M^{2}\right)^{3}} \\
& -\frac{k^{6} \Lambda^{4}}{140\left(\Lambda^{2}+M^{2}\right)^{4}}-\frac{k^{8} \Lambda^{4}}{630\left(\Lambda^{2}+M^{2}\right)^{5}}+\cdots,
\end{aligned}
$$

which would vanish if the amplitude were gauge invariant. Further,

$$
\Pi_{\mu \nu}^{(0)}=\Pi_{\mu \nu}(\Lambda ; 0)=i \frac{e^{2} Q^{2} N_{c} N_{f}}{8 \pi^{2}} g_{\mu \nu} \mathcal{A}_{g}(\Lambda ; 0)=-i \frac{e^{2} Q^{2} N_{c} N_{f}}{8 \pi^{2}} g_{\mu \nu} \Lambda^{4} /\left(\Lambda^{2}+M^{2}\right) .
$$

\footnotetext{
${ }^{8}$ We impose that the on-shell conditions (except for the self-energy) and the momentum conservation for the external momenta are satisfied.
} 

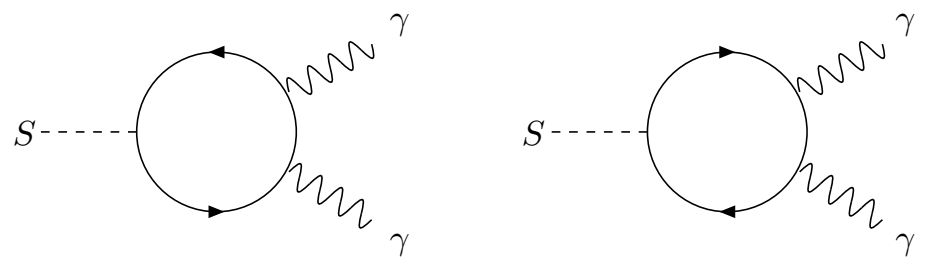

Figure 8. $S-\gamma-\gamma$ coupling.

This defines the tensor structure for the counter terms, because this term is not gauge invariant and has to be subtracted. Therefore, the subtracted amplitude is $\mathcal{A}_{g}^{R}\left(\Lambda ; k^{2}\right)=$ $\mathcal{A}_{g}\left(\Lambda ; k^{2}\right)-\mathcal{G}\left(\Lambda ; k^{2}\right)$. Obviously, in this example, all the non-gauge invariant terms can be canceled by the counter terms of this tensor structure. That is, no more new tensor structure is needed for the counter terms.

Since in this example we know the closed expression for the amplitude, it is not necessary to implement least subtraction procedure. As we see from (A.3), the $\Lambda^{4} g_{\mu \nu}$ term is not gauge invariant. This non-gauge invariant term, which is a photon mass function $\Pi_{\mu \nu}^{\Lambda}(\Lambda ; k)$, can not be made gauge invariant by adding $k_{\mu} k_{\nu}$ terms without introducing a singularity in $k^{2}$. Therefore, we have to subtract $\Pi_{\mu \nu}^{\Lambda}(\Lambda ; k)$ from the self-energy $\Pi_{\mu \nu}(\Lambda ; k)$, in accord with least subtraction procedure as described above.

The gauge invariant term proportional $\propto \Lambda^{2}$ in (A.3) gives a wrong normalization so that further counter terms are needed. Finally, we have the normalized subtracted gauge invariant self-energy of the photon:

$$
\begin{aligned}
\Pi_{\mu \nu}^{R}(\Lambda ; k)= & i \frac{e^{2} Q^{2} N_{c} N_{f}}{8 \pi^{2}}\left(g_{\mu \nu} k^{2}-k_{\mu} k_{\nu}\right) \int_{0}^{1} d x\left[\left(\frac{4 \Lambda^{2}(1-x) x}{\Lambda^{2}+B^{2}}-\frac{2 \Lambda^{2} / 3}{\Lambda^{2}+M^{2}}\right)\right. \\
& \left.\left.\left.+4(1-x) x\left\{\ln \left(1-\frac{(1-x) x k^{2}}{M^{2}}\right)\right)-\ln \left(1-\frac{(1-x) x k^{2}}{\Lambda^{2}+M^{2}}\right)\right)\right\}\right] .
\end{aligned}
$$

- $\boldsymbol{S}-\boldsymbol{\gamma}-\boldsymbol{\gamma}$ amplitude. The next example is the $S(p)-\gamma(k)-\gamma\left(k^{\prime}\right)$ three-point function. There are two diagrams at the one-loop level as shown in figure 8. Again using the usual technique, we obtain the unsubtracted amplitude

$$
\begin{aligned}
\mathcal{A}_{\mu \nu}\left(\Lambda ; k, k^{\prime}\right)= & i \frac{e^{2} Q^{2} y N_{c} N_{f} M}{4 \pi^{2}} \int_{0}^{1} d x \int_{0}^{1-x} d y \frac{2 \Lambda^{4}}{\left(\Lambda^{2}+D^{2}\right)^{2}} \\
& \times\left[g_{\mu \nu}-\frac{(1-4 x y)\left(g_{\mu \nu} k \cdot k^{\prime}-k_{\mu} k_{\nu}^{\prime}\right)}{D^{2}}\right],
\end{aligned}
$$

where

$$
D^{2}=M^{2}-2 x y k \cdot k^{\prime} .
$$

The last term in the square bracket has an imaginary part and gauge invariant. The first $g_{\mu \nu}$ term in the square bracket is not gauge invariant. It is obvious that least subtraction 
procedure implies a complete subtraction of this term. The subtracted amplitude is

$$
\mathcal{A}_{\mu \nu}^{R}\left(\Lambda ; k, k^{\prime}\right)=-i \frac{e^{2} Q^{2} y N_{c} N_{f} M}{4 \pi^{2}} \int_{0}^{1} d x \int_{0}^{1-x} d y \frac{2 \Lambda^{4}}{\left(\Lambda^{2}+D^{2}\right)^{2}} \frac{(1-4 x y)\left(g_{\mu \nu} k \cdot k^{\prime}-k_{\mu} k_{\nu}^{\prime}\right)}{D^{2}} .
$$

- $\phi-\phi-\gamma-\gamma$ amplitude. The next example is the $\phi(p)-\phi\left(p^{\prime}\right)-\gamma(k)-\gamma\left(k^{\prime}\right)$ four-point function. The diagrams at the one-loop level are shown in figure $3 \sim 5$ with the sum of the unsubtracted amplitudes:

$$
\mathcal{A}_{\mu \nu}\left(\Lambda ; k, k^{\prime}, p, p^{\prime}\right)=\mathcal{A}_{\mu \nu}^{(a)}\left(\Lambda ; k, k^{\prime}, p, p^{\prime}\right)+\mathcal{A}_{\mu \nu}^{(b)}\left(\Lambda ; k, k^{\prime}, p, p^{\prime}\right)+\mathcal{A}_{\mu \nu}^{(c)}\left(\Lambda ; k, k^{\prime}, p, p^{\prime}\right) .
$$

The diagrams of figure 3 , which give $\mathcal{A}_{\mu \nu}^{(a)}$ in (A.10), are simpler to compute, because the structure is the same as figure 8. Again using the usual technique, we obtain the unsubtracted amplitude (with $N_{f}=3$ )

$$
\begin{aligned}
\mathcal{A}_{\mu \nu}^{(a)}\left(\Lambda ; k, k^{\prime}\right)= & -i\left[\frac{e^{2} Q^{2} N_{c}}{2 \pi^{2}}\right]\left[\frac{G_{D} M}{8 G^{2}}\right] \int_{0}^{1} d x \int_{0}^{1-x} d y \\
& \times \frac{2 \Lambda^{4}}{\left(\Lambda^{2}+D^{2}\right)^{2}}\left[g_{\mu \nu}-\frac{(1-4 x y)\left(g_{\mu \nu} k \cdot k^{\prime}-k_{\mu} k_{\nu}^{\prime}\right)}{D^{2}}\right],
\end{aligned}
$$

where $D^{2}$ is given in (A.8). The non-gauge invariant terms have the same structure as (A.7). Therefore, we subtract the non-gauge invariant $g_{\mu \nu}$ term:

$$
\mathcal{A}_{\mu \nu}^{R(a)}=\left(g_{\mu \nu} k \cdot k^{\prime}-k_{\mu} k_{\nu}^{\prime}\right) \mathcal{A}^{R(a)}=\mathcal{A}_{\mu \nu}^{(a)} \text { with the first } g_{\mu \nu} \text { term omitted. }
$$

The tensor structure of the other diagrams is more complicated. The amplitude has to satisfy the Bose symmetry:

$$
\mathcal{A}_{\mu \nu}\left(\Lambda ; k, k^{\prime}, p, p^{\prime}\right)=\mathcal{A}_{\nu \mu}\left(\Lambda ; k^{\prime}, k, p, p^{\prime}\right) \text { and } \mathcal{A}_{\mu \nu}\left(\Lambda ; k, k^{\prime}, p, p^{\prime}\right)=\mathcal{A}_{\mu \nu}\left(\Lambda ; k, k^{\prime}, p^{\prime}, p\right) .
$$

To proceed we introduce the Mandelstam variables:

$$
\begin{aligned}
& S=\left(p+p^{\prime}\right)^{2}=2 m_{\mathrm{DM}}^{2}+2 p \cdot p^{\prime}=\left(k+k^{\prime}\right)^{2}=2 k \cdot k^{\prime}, \\
& T=(p-k)^{2}=m_{\mathrm{DM}}^{2}-2 p \cdot k, U=\left(p-k^{\prime}\right)^{2}=m_{\mathrm{DM}}^{2}-2 p \cdot k^{\prime} .
\end{aligned}
$$

All the dot products of the momenta and $m_{\mathrm{DM}}^{2}$ can be expressed as a function of $S, T$ and $U$. The most general tensor structure, which is consistent with the Bose symmetry (A.13) is ${ }^{9}$

$$
\begin{aligned}
\mathcal{A}_{\mu \nu}^{(b+c)}\left(\Lambda ; k, k^{\prime}, p, p^{\prime}\right)= & \mathcal{A}_{\mu \nu}^{(b)}\left(\Lambda ; k, k^{\prime}, p, p^{\prime}\right)+\mathcal{A}_{\mu \nu}^{(c)}\left(\Lambda ; k, k^{\prime}, p, p^{\prime}\right) \\
= & i\left[\frac{e^{2} Q^{2} N_{c}}{2 \pi^{2}}\right]\left(1-\frac{G_{D}\langle\sigma\rangle}{8 G^{2}}\right)^{2}\left\{g_{\mu \nu} \mathcal{A}_{g}(\Lambda ; S, T, U)\right. \\
& +k_{\mu} k_{\nu}^{\prime} \mathcal{A}_{k k}(\Lambda ; S, T, U)+\left(p_{\mu} p_{\nu}+p_{\mu}^{\prime} p_{\nu}^{\prime}\right) \mathcal{A}_{p}(\Lambda ; S, T, U) \\
& +p_{\mu}^{\prime} p_{\nu} \mathcal{A}_{p^{\prime}}(\Lambda ; S, T, U)+p_{\mu} p_{\nu}^{\prime} \mathcal{A}_{p^{\prime}}(\Lambda ; S, U, T) \\
& \left.+\left(k_{\mu} p_{\nu}^{\prime}+p_{\mu} k_{\nu}^{\prime}\right) \mathcal{A}_{k}(\Lambda ; S, T, U)+\left(k_{\mu} p_{\nu}+p_{\mu}^{\prime} k_{\nu}^{\prime}\right) \mathcal{A}_{k}(\Lambda ; S, U, T)\right\},
\end{aligned}
$$

\footnotetext{
${ }^{9}$ Because of the on-shell gauge invariance, we have suppressed terms proportional to $k_{\nu}$ and $k_{\mu}^{\prime}$ in (A.16).
} 
where the amplitudes $\mathcal{A}_{i}$ 's have to satisfy

$$
\mathcal{A}_{g, k k, p}(\Lambda ; S, T, U)=\mathcal{A}_{g, k k, p}(\Lambda ; S, U, T)
$$

Gauge invariance means that the following quantities vanish:

$$
\begin{aligned}
k^{\nu} \mathcal{A}_{\mu \nu}\left(\Lambda ; k, k^{\prime}, p, p^{\prime}\right) & =k_{\mu} \mathcal{G}_{1}(\Lambda ; S, T, U)+p_{\mu} \mathcal{G}_{2}(\Lambda ; S, T, U)+p_{\mu}^{\prime} \mathcal{G}_{3}(\Lambda ; S, T, U), \\
k^{\prime \mu} \mathcal{A}_{\mu \nu}\left(\Lambda ; k, k^{\prime}, p, p^{\prime}\right) & =k_{\nu}^{\prime} \mathcal{G}_{1}^{\prime}(\Lambda ; S, T, U)+p_{\nu} \mathcal{G}_{2}^{\prime}(\Lambda ; S, T, U)+p_{\nu}^{\prime} \mathcal{G}_{3}^{\prime}(\Lambda ; S, T, U),
\end{aligned}
$$

where we impose the on-shell condition $k^{2}=k^{\prime 2}=0, p^{2}=p^{2}=m_{\mathrm{DM}}^{2}$ along with the four momentum conservation $p+p^{\prime}=k+k^{\prime}$, and $\mathcal{G}_{i}$ 's are defined as

$$
\begin{aligned}
& \mathcal{G}_{1}(\Lambda ; S, T, U)=\mathcal{G}_{1}^{\prime}(\Lambda ; S, T, U) \\
& \quad=\mathcal{A}_{g}(\Lambda ; S, T, U)+\frac{S}{4}\left[2 \mathcal{A}_{k k}(\Lambda ; S, T, U)+\mathcal{A}_{k}(\Lambda ; S, T, U)+\mathcal{A}_{k}(\Lambda ; S, U, T)\right] \\
& \quad+\frac{1}{4}(T-U)\left[\mathcal{A}_{k}(\Lambda ; S, T, U)-\mathcal{A}_{k}(\Lambda ; S, U, T)\right] \\
& \mathcal{G}_{2}(\Lambda ; S, T, U)=\mathcal{G}_{3}^{\prime}(\Lambda ; S, T, U)=\mathcal{G}_{3}(\Lambda ; S, U, T) \\
& \quad=\frac{1}{4}(S-T+U) \mathcal{A}_{p}(\Lambda ; S, T, U)+\frac{S}{2} \mathcal{A}_{k}(\Lambda ; S, T, U)+\frac{1}{4}(S+T-U) \mathcal{A}_{p^{\prime}}(\Lambda ; S, U, T), \\
& \mathcal{G}_{3}(\Lambda ; S, T, U)=\mathcal{G}_{2}^{\prime}(\Lambda ; S, T, U)=\mathcal{G}_{2}(\Lambda ; S, U, T) \\
& \quad=\frac{1}{4}(S+T-U) \mathcal{A}_{p}(\Lambda ; S, U, T)+\frac{S}{2} \mathcal{A}_{k}(\Lambda ; S, U, T)+\frac{1}{4}(S-T+U) \mathcal{A}_{p^{\prime}}(\Lambda ; S, T, U) .
\end{aligned}
$$

Gauge invariance requires that all $\mathcal{G}_{i}$ 's should vanish identically. We have calculated them for $\mathcal{A}_{\mu \nu}^{(b+c)}=\mathcal{A}_{\mu \nu}^{(b)}+\mathcal{A}_{\mu \nu}^{(c)}$ explicitly for a small external momenta $\sim \mathcal{O}(\epsilon)$ and find

$$
\begin{aligned}
\epsilon=0: \mathcal{G}_{1}= & \frac{\Lambda^{4}}{\left(\Lambda^{2}+M^{2}\right)^{2}}, \quad \mathcal{G}_{2}=0, \\
\epsilon^{2}: \mathcal{G}_{1}= & \frac{11 \Lambda^{4}}{40\left(\Lambda^{2}+M^{2}\right)^{3}}[S+2(T+U)], \mathcal{G}_{2}=\frac{3 \Lambda^{4}}{40\left(\Lambda^{2}+M^{2}\right)^{3}}(T-U), \\
\epsilon^{4}: \mathcal{G}_{1}= & \frac{\Lambda^{4}}{280\left(\Lambda^{2}+M^{2}\right)^{4}}\left[82 S^{2}+275 S(T+U)+64\left\{4\left(T^{2}+U^{2}\right)+7 T U\right\}\right], \\
\mathcal{G}_{2}= & \frac{3 \Lambda^{4}}{280\left(\Lambda^{2}+M^{2}\right)^{4}}(T-U)[16 S+13(T+U)], \\
\epsilon^{6}: \mathcal{G}_{1}= & \frac{\Lambda^{4}}{1008\left(\Lambda^{2}+M^{2}\right)^{5}}\left[517 S^{3}+2168 S^{2}(T+U)+10 S\left\{337\left(T^{2}+U^{2}\right)+586 T U\right\}\right. \\
& \left.+3\left\{761\left(T^{3}+U^{3}\right)+1591\left(T^{2} U+T U^{2}\right)\right\}\right], \\
\mathcal{G}_{2}= & \frac{\Lambda^{4}}{336\left(\Lambda^{2}+M^{2}\right)^{5}}(T-U)\left[116 S^{2}+239 S(T+U)+17\left\{7\left(T^{2}+U^{2}\right)+10 T U\right\}\right], \\
\epsilon^{8}: \mathcal{G}_{1}= & \frac{\Lambda^{4}}{1584\left(\Lambda^{2}+M^{2}\right)^{6}}\left[2142 S^{4}+10058 S^{3}(T+U)+2 S^{2}\left(\left\{9701\left(T^{2}+U^{2}\right)+17284 T U\right\}\right.\right. \\
& +15 S\left\{1399\left(T^{3}+U^{3}\right)+3137\left(T^{2} U+T U^{2}\right)\right\}+3\left\{3993\left(T^{4}+U^{4}\right)\right. \\
& \left.\left.+9178\left(T^{3} U+T U^{3}\right)+11098 T^{2} U^{2}\right\}\right],
\end{aligned}
$$




$$
\begin{aligned}
\mathcal{G}_{2}= & \frac{\Lambda^{4}}{1584\left(\Lambda^{2}+M^{2}\right)^{6}}(T-U)\left[1512 S^{3}+4007 S^{2}(T+U)\right. \\
& \left.+15 S\left\{319\left(T^{2}+U^{2}\right)+512 T U\right\}+630\left\{3\left(T^{3}+U^{3}\right)+5\left(T^{2} U+T U^{2}\right)\right\}\right] .
\end{aligned}
$$

As we see from $\left(\right.$ A.23) $\sim\left(\right.$ A.27) that the non-gauge invariant function $\mathcal{G}_{1}$ has the same structure as $\mathcal{G}$ in (A.4) for the photon self-energy. Therefore, we subtract this term from the amplitude so that the function $\mathcal{A}_{g}$ is replaced by

$$
\mathcal{A}_{g}^{R}=\mathcal{A}_{g}-\mathcal{G}_{1}
$$

where $\mathcal{A}_{g}$ and $\mathcal{G}_{1}$ are defined in (A.16) and (A.20), respectively. At $\mathcal{O}\left(\epsilon^{2}\right) \mathcal{G}_{2}$ becomes non-zero. As we see from (A.18) and (A.19), this non-gauge invariant term requires an introduction of a new tensor structure for counter terms. We see from (A.23) (A.27) that $\mathcal{G}_{2}$ is proportional to $(T-U)$, so that we can rewrite it as

$$
\mathcal{G}_{2}(\Lambda ; S, T, U)=(T-U) \hat{\mathcal{G}}_{2}(\Lambda ; S, T, U)
$$

which will be justified below to all orders in the expansion of $k^{\prime}$ s and $p^{\prime}$ s. Therefore, we can cancel this non-gauge invariant term by adding counter terms such that $\mathcal{A}_{p}$ and $\mathcal{A}_{p^{\prime}}$ change according to

$$
\mathcal{A}_{p} \rightarrow \mathcal{A}_{p}^{R}=\mathcal{A}_{p}+2 \hat{\mathcal{G}_{2}}, \mathcal{A}_{p^{\prime}} \rightarrow \mathcal{A}_{p^{\prime}}^{R}=\mathcal{A}_{p^{\prime}}-2 \hat{\mathcal{G}_{2}},
$$

where $\mathcal{A}_{p}$ and $\mathcal{A}_{p^{\prime}}$ are defined in (A.16). Since $\mathcal{A}_{p}$ has to satisfy the Bose symmetry (A.17), $\hat{\mathcal{G}}_{2}$, too, has to satisfy the same symmetry. We have numerically checked that

$$
\mathcal{G}_{2}(\Lambda ; S, T, T)=0, \mathcal{G}_{2}(\Lambda ; S, T, U)=-\mathcal{G}_{2}(\Lambda ; S, U, T)
$$

is satisfied within an accuracy that we can get, which implies that $\hat{\mathcal{G}}_{2}(\Lambda ; S, T, U)=$ $\hat{\mathcal{G}}_{2}(\Lambda ; S, U, T)$.

Finally, we have the gauge invariant $\phi-\phi-\gamma-\gamma$ four-point function:

$$
\begin{aligned}
\mathcal{A}_{\mu \nu}^{R}\left(\Lambda ; k, k^{\prime}, p, p^{\prime}\right)= & \mathcal{A}_{\mu \nu}^{R(a)}\left(\Lambda ; k, k^{\prime}, p, p^{\prime}\right)+i\left[\frac{e^{2} Q^{2} N_{c}}{2 \pi^{2}}\right]\left(1-\frac{G_{D}\langle\sigma\rangle}{8 G^{2}}\right)^{2}\left\{g_{\mu \nu} \mathcal{A}_{g}^{R}(\Lambda ; S, T, U)\right. \\
& +k_{\mu} k_{\nu}^{\prime} \mathcal{A}_{k k}(\Lambda ; S, T, U)+\left(p_{\mu} p_{\nu}+p_{\mu}^{\prime} p_{\nu}^{\prime}\right) \mathcal{A}_{p}^{R}(\Lambda ; S, T, U) \\
& +p_{\mu}^{\prime} p_{\nu} \mathcal{A}_{p^{\prime}}^{R}(\Lambda ; S, T, U)+p_{\mu} p_{\nu}^{\prime} \mathcal{A}_{p^{\prime}}^{R}(\Lambda ; S, U, T) \\
& \left.+\left(k_{\mu} p_{\nu}^{\prime}+p_{\mu} k_{\nu}^{\prime}\right) \mathcal{A}_{k}(\Lambda ; S, T, U)+\left(k_{\mu} p_{\nu}+p_{\mu}^{\prime} k_{\nu}^{\prime}\right) \mathcal{A}_{k}(\Lambda ; S, U, T)\right\},(\mathrm{A} .32
\end{aligned}
$$

where $\mathcal{A}^{R(a)}$ is given in (A.12). Note that for the case $T=U$ the function $\mathcal{G}_{2}$ vanishes. In this case, therefore, all the counter terms are proportional to the metric tensor. In the center of mass system, $T=U$ implies $p=p^{\prime}=\left(m_{\mathrm{DM}}, \mathbf{0}\right)$, and only $k$ and $k^{\prime}$ are independent Lorentz vectors $\left(p_{\mu}=\left(k_{\mu}+k_{\mu}^{\prime}\right) / 2\right)$. In this system, the non-gauge invariant terms of $\mathcal{A}_{\mu \nu}$ are proportional to $g_{\mu \nu}$. No Lorentz transformation can produce non-gauge invariant terms proportional to $k_{\mu} p_{\nu}$ or $p_{\mu} p_{\nu}$ from the $g_{\mu \nu}$ term. This is the reason why $\mathcal{G}_{2}$ vanishes when $T=U$, and therefore the assumption that this function takes the form $\mathcal{G}_{2}=(T-U) \hat{\mathcal{G}}_{2}$ is 
justified to all orders in the expansion of $k^{\prime} \mathrm{s}$ and $p^{\prime}$ s. The amplitude with $p=p^{\prime}=\left(m_{\mathrm{DM}}, \mathbf{0}\right)$ will be used for the annihilation of two DM's into two photons in section V.

The tensor structure (A.16) is the most general one. However, using the on-shell conditions $k+k^{\prime}=p+p^{\prime}$ we can eliminate $k$ and $k^{\prime}$, because $k_{\nu}$ and $k_{\mu}^{\prime}$ terms do not contribute neither to the physical amplitude nor to the on-shell gauge invariance conditions (A.18) and (A.19) and hence can be suppressed as we have done in (A.16). In this basis, $\mathcal{A}_{\mu \nu}^{(b+c)}\left(\Lambda ; k, k^{\prime}, p, p^{\prime}\right)$ of (A.16) becomes

$$
\begin{aligned}
\mathcal{A}_{\mu \nu}^{(b+c)}\left(\Lambda ; k, k^{\prime}, p, p^{\prime}\right)= & i\left[\frac{e^{2} Q^{2} N_{c}}{2 \pi^{2}}\right]\left(1-\frac{G_{D}\langle\sigma\rangle}{8 G^{2}}\right)^{2} \\
\times & \left\{g_{\mu \nu} \mathcal{A}_{g}(\Lambda ; S, T, U)+\left(p_{\mu} p_{\nu}+p_{\mu}^{\prime} p_{\nu}^{\prime}\right) \tilde{\mathcal{A}}_{p}(\Lambda ; S, T, U)\right. \\
& \left.+p_{\mu}^{\prime} p_{\nu} \tilde{\mathcal{A}}_{p^{\prime}}(\Lambda ; S, T, U)+p_{\mu} p_{\nu}^{\prime} \tilde{\mathcal{A}}_{p^{\prime}}(\Lambda ; S, U, T)\right\}
\end{aligned}
$$

where

$$
\begin{aligned}
\tilde{\mathcal{A}}_{p}(\Lambda ; S, T, U)= & \mathcal{A}_{p}(\Lambda ; S, T, U)+\mathcal{A}_{k k}(\Lambda ; S, T, U)+\mathcal{A}_{k}(\Lambda ; S, T, U) \\
& +\mathcal{A}_{k}(\Lambda ; S, U, T) \\
\tilde{\mathcal{A}}_{p^{\prime}}(\Lambda ; S, T, U)= & \mathcal{A}_{p^{\prime}}(\Lambda ; S, T, U)+\mathcal{A}_{k k}(\Lambda ; S, T, U)+2 \mathcal{A}_{k}(\Lambda ; S, U, T) .
\end{aligned}
$$

Then the gauge invariance conditions (A.18) and (A.19) mean that

$$
\begin{aligned}
\tilde{\mathcal{G}}_{2}(\Lambda ; S, T, U) & =\tilde{\mathcal{G}}_{3}(\Lambda ; S, U, T) \\
& =\frac{1}{4}(S-T+U) \tilde{\mathcal{A}}_{p}(\Lambda ; S, T, U)+\frac{1}{4}(S+T-U) \tilde{\mathcal{A}}_{p^{\prime}}(\Lambda ; S, U, T) \\
& =\mathcal{G}_{1}(\Lambda ; S, T, U)+(T-U) \hat{\mathcal{G}}_{2}(\Lambda ; S, T, U)
\end{aligned}
$$

has to vanish, where $\mathcal{G}_{1}$ and $\hat{\mathcal{G}}_{2}$ are defined in (A.20) and (A.29), respectively. From (A.36) and (A.37) it is now obvious how to restore gauge invariance: from (A.37) we see that $\tilde{\mathcal{G}}_{2}(\Lambda ; S, T, U)$ can be uniquely divided into the even and odd part under the interchange $T \leftrightarrow U$, because $\mathcal{G}_{1}$ and $\hat{\mathcal{G}}_{2}$ are even functions. The odd part can be canceled by

$$
\tilde{\mathcal{A}}_{p} \rightarrow \tilde{\mathcal{A}}_{p}^{R}=\tilde{\mathcal{A}}_{p}+2 \hat{\mathcal{G}}_{2}, \tilde{\mathcal{A}}_{p^{\prime}} \rightarrow \tilde{\mathcal{A}}_{p^{\prime}}^{R}=\tilde{\mathcal{A}}_{p^{\prime}}-2 \hat{\mathcal{G}}_{2},
$$

as in the case of $\mathcal{A}_{p}$ and $\mathcal{A}_{p^{\prime}}$ (see (A.30)). The even part can be canceled by the redefinition of $A_{g}$ which is defined in (A.28). The resulting gauge invariant subtracted amplitude is identical with (A.32) up to on-shell conditions.

Open Access. This article is distributed under the terms of the Creative Commons Attribution License (CC-BY 4.0), which permits any use, distribution and reproduction in any medium, provided the original author(s) and source are credited.

\section{References}

[1] ATLAS collaboration, Observation of a new particle in the search for the Standard Model Higgs boson with the ATLAS detector at the LHC, Phys. Lett. B 716 (2012) 1 [arXiv: 1207.7214] [INSPIRE]. 
[2] CMS collaboration, Observation of a new boson at a mass of $125 \mathrm{GeV}$ with the CMS experiment at the LHC, Phys. Lett. B 716 (2012) 30 [arXiv:1207.7235] [INSPIRE].

[3] M. Holthausen, K.S. Lim and M. Lindner, Planck scale boundary conditions and the Higgs mass, JHEP 02 (2012) 037 [arXiv:1112.2415] [INSPIRE].

[4] G. Degrassi et al., Higgs mass and vacuum stability in the Standard Model at NNLO, JHEP 08 (2012) 098 [arXiv: 1205.6497] [INSPIRE].

[5] D. Buttazzo et al., Investigating the near-criticality of the Higgs boson, JHEP 12 (2013) 089 [arXiv: 1307.3536] [INSPIRE].

[6] F. Bezrukov, M.Y. Kalmykov, B.A. Kniehl and M. Shaposhnikov, Higgs boson mass and new physics, JHEP 10 (2012) 140 [arXiv:1205.2893] [INSPIRE].

[7] J. Kubo, K.S. Lim and M. Lindner, Electroweak symmetry breaking by QCD, arXiv: 1403.4262 [INSPIRE].

[8] S.R. Coleman and E.J. Weinberg, Radiative corrections as the origin of spontaneous symmetry breaking, Phys. Rev. D 7 (1973) 1888 [INSPIRE].

[9] J.P. Fatelo, J.M. Gerard, T. Hambye and J. Weyers, Symmetry breaking induced by top loops, Phys. Rev. Lett. 74 (1995) 492 [INSPIRE].

[10] R. Hempfling, The next-to-minimal Coleman-Weinberg model, Phys. Lett. B 379 (1996) 153 [hep-ph/9604278] [INSPIRE].

[11] T. Hambye, Symmetry breaking induced by top quark loops from a model without scalar mass, Phys. Lett. B 371 (1996) 87 [hep-ph/9510266] [INSPIRE].

[12] K.A. Meissner and H. Nicolai, Conformal symmetry and the Standard Model, Phys. Lett. B 648 (2007) 312 [hep-th/0612165] [INSPIRE].

[13] K.A. Meissner and H. Nicolai, Effective action, conformal anomaly and the issue of quadratic divergences, Phys. Lett. B 660 (2008) 260 [arXiv:0710.2840] [INSPIRE].

[14] K.A. Meissner and H. Nicolai, Conformal invariance from non-conformal gravity, Phys. Rev. D 80 (2009) 086005 [arXiv:0907.3298] [INSPIRE].

[15] R. Foot, A. Kobakhidze and R.R. Volkas, Electroweak Higgs as a pseudo-Goldstone boson of broken scale invariance, Phys. Lett. B 655 (2007) 156 [arXiv:0704.1165] [INSPIRE].

[16] R. Foot, A. Kobakhidze and R.R. Volkas, Cosmological constant in scale-invariant theories, Phys. Rev. D 84 (2011) 075010 [arXiv: 1012.4848] [INSPIRE].

[17] R. Foot, A. Kobakhidze, K. McDonald and R. Volkas, Neutrino mass in radiatively-broken scale-invariant models, Phys. Rev. D 76 (2007) 075014 [arXiv:0706.1829] [inSPIRE].

[18] R. Foot, A. Kobakhidze, K.L. McDonald and R.R. Volkas, A solution to the hierarchy problem from an almost decoupled hidden sector within a classically scale invariant theory, Phys. Rev. D 77 (2008) 035006 [arXiv:0709.2750] [InSPIRE].

[19] R. Foot, A. Kobakhidze, K.L. McDonald and R.R. Volkas, Poincaré protection for a natural electroweak scale, Phys. Rev. D 89 (2014) 115018 [arXiv: 1310.0223] [INSPIRE].

[20] W.-F. Chang, J.N. Ng and J.M.S. Wu, Shadow Higgs from a scale-invariant hidden U(1)s model, Phys. Rev. D 75 (2007) 115016 [hep-ph/0701254] [INSPIRE].

[21] T. Hambye and M.H.G. Tytgat, Electroweak symmetry breaking induced by dark matter, Phys. Lett. B 659 (2008) 651 [arXiv:0707.0633] [INSPIRE]. 
[22] S. Iso, N. Okada and Y. Orikasa, Classically conformal B-L extended Standard Model, Phys. Lett. B 676 (2009) 81 [arXiv: 0902.4050] [INSPIRE].

[23] S. Iso, N. Okada and Y. Orikasa, The minimal B-L model naturally realized at TeV scale, Phys. Rev. D 80 (2009) 115007 [arXiv:0909.0128] [InSPIRE].

[24] S. Iso and Y. Orikasa, TeV scale B-L model with a flat Higgs potential at the Planck scale in view of the hierarchy problem, Prog. Theor. Exp. Phys. 2013 (2013) $023 \mathrm{~B} 08$ [arXiv: 1210.2848] [INSPIRE].

[25] M. Holthausen, M. Lindner and M.A. Schmidt, Radiative symmetry breaking of the minimal left-right symmetric model, Phys. Rev. D 82 (2010) 055002 [arXiv:0911.0710] [InSPIRE].

[26] K. Ishiwata, Dark matter in classically scale-invariant two singlets Standard Model, Phys. Lett. B 710 (2012) 134 [arXiv:1112.2696] [INSPIRE].

[27] V.V. Khoze, Inflation and dark matter in the Higgs portal of classically scale invariant Standard Model, JHEP 11 (2013) 215 [arXiv:1308.6338] [INSPIRE].

[28] Y. Kawamura, Naturalness, conformal symmetry and duality, Prog. Theor. Exp. Phys. 2013 (2013) 113B04 [arXiv:1308.5069] [INSPIRE].

[29] F. Gretsch and A. Monin, Dilaton: saving conformal symmetry, arXiv:1308.3863 [INSPIRE].

[30] C.D. Carone and R. Ramos, Classical scale-invariance, the electroweak scale and vector dark matter, Phys. Rev. D 88 (2013) 055020 [arXiv:1307.8428] [INSPIRE].

[31] V.V. Khoze and G. Ro, Leptogenesis and neutrino oscillations in the classically conformal Standard Model with the Higgs portal, JHEP 10 (2013) 075 [arXiv:1307.3764] [INSPIRE].

[32] E. Gabrielli et al., Towards completing the Standard Model: vacuum stability, EWSB and dark matter, Phys. Rev. D 89 (2014) 015017 [arXiv: 1309.6632] [InSPIRE].

[33] C. Englert, J. Jaeckel, V.V. Khoze and M. Spannowsky, Emergence of the electroweak scale through the Higgs portal, JHEP 04 (2013) 060 [arXiv:1301.4224] [INSPIRE].

[34] A. Farzinnia, H.-J. He and J. Ren, Natural electroweak symmetry breaking from scale invariant Higgs mechanism, Phys. Lett. B 727 (2013) 141 [arXiv:1308.0295] [INSPIRE].

[35] S. Abel and A. Mariotti, Novel Higgs potentials from gauge mediation of exact scale breaking, arXiv:1312.5335 [INSPIRE].

[36] M. Ibe, S. Matsumoto and T.T. Yanagida, Flat Higgs potential from Planck scale supersymmetry breaking, Phys. Lett. B 732 (2014) 214 [arXiv:1312.7108] [INSPIRE].

[37] C.T. Hill, Is the Higgs boson associated with Coleman-Weinberg dynamical symmetry breaking?, Phys. Rev. D 89 (2014) 073003 [arXiv:1401.4185] [INSPIRE].

[38] J. Guo and Z. Kang, Higgs naturalness and dark matter stability by scale invariance, arXiv: 1401.5609 [INSPIRE].

[39] S. Benic and B. Radovcic, Electroweak breaking and dark matter from the common scale, Phys. Lett. B 732 (2014) 91 [arXiv: 1401.8183] [inSPIRE].

[40] V.V. Khoze, C. McCabe and G. Ro, Higgs vacuum stability from the dark matter portal, JHEP 08 (2014) 026 [arXiv: 1403.4953] [INSPIRE].

[41] H. Davoudiasl and I.M. Lewis, Right-handed neutrinos as the origin of the electroweak scale, Phys. Rev. D 90 (2014) 033003 [arXiv:1404.6260] [INSPIRE]. 
[42] P.H. Chankowski, A. Lewandowski, K.A. Meissner and H. Nicolai, Softly broken conformal symmetry and the stability of the electroweak scale, arXiv:1404.0548 [INSPIRE].

[43] C.G. Callan Jr., Broken scale invariance in scalar field theory, Phys. Rev. D 2 (1970) 1541 [INSPIRE].

[44] K. Symanzik, Small distance behavior in field theory and power counting, Commun. Math. Phys. 18 (1970) 227 [INSPIRE].

[45] W.A. Bardeen, On naturalness in the Standard Model, FERMILAB-CONF-95-391, Fermilab, Batavia U.S.A. (1995).

[46] T. Hur, D.-W. Jung, P. Ko and J.Y. Lee, Electroweak symmetry breaking and cold dark matter from strongly interacting hidden sector, Phys. Lett. B 696 (2011) 262 [arXiv:0709.1218] [INSPIRE].

[47] T. Hur and P. Ko, Scale invariant extension of the Standard Model with strongly interacting hidden sector, Phys. Rev. Lett. 106 (2011) 141802 [arXiv:1103.2571] [INSPIRE].

[48] M. Heikinheimo, A. Racioppi, M. Raidal, C. Spethmann and K. Tuominen, Physical naturalness and dynamical breaking of classical scale invariance, Mod. Phys. Lett. A 29 (2014) 1450077 [arXiv:1304.7006] [INSPIRE].

[49] M. Holthausen, J. Kubo, K.S. Lim and M. Lindner, Electroweak and conformal symmetry breaking by a strongly coupled hidden sector, JHEP 12 (2013) 076 [arXiv:1310.4423] [INSPIRE].

[50] M.J. Strassler and K.M. Zurek, Echoes of a hidden valley at hadron colliders, Phys. Lett. B 651 (2007) 374 [hep-ph/0604261] [INSPIRE].

[51] T. Han, Z. Si, K.M. Zurek and M.J. Strassler, Phenomenology of hidden valleys at hadron colliders, JHEP 07 (2008) 008 [arXiv:0712.2041] [INSPIRE].

[52] T. Bringmann, L. Bergstrom and J. Edsjo, New gamma-ray contributions to supersymmetric dark matter annihilation, JHEP 01 (2008) 049 [arXiv:0710.3169] [INSPIRE].

[53] G. Bertone, C.B. Jackson, G. Shaughnessy, T.M.P. Tait and A. Vallinotto, The WIMP forest: indirect detection of a chiral square, Phys. Rev. D 80 (2009) 023512 [arXiv: 0904.1442] [INSPIRE].

[54] R. Laha, K.C.Y. Ng, B. Dasgupta and S. Horiuchi, Galactic center radio constraints on gamma-ray lines from dark matter annihilation, Phys. Rev. D 87 (2013) 043516 [arXiv: 1208.5488] [INSPIRE].

[55] LAT collaboration, M. Ackermann et al., Fermi LAT search for dark matter in gamma-ray lines and the inclusive photon spectrum, Phys. Rev. D 86 (2012) 022002 [arXiv:1205.2739] [INSPIRE].

[56] Fermi-LAT collaboration, M. Gustafsson, Fermi-LAT and the gamma-ray line search, arXiv:1310.2953 [INSPIRE].

[57] H.E.S.S. collaboration, A. Abramowski et al., Search for photon line-like signatures from dark matter annihilations with H.E.S.S., Phys. Rev. Lett. 110 (2013) 041301 [arXiv: 1301.1173] [INSPIRE].

[58] E. Bulbul et al., Detection of an unidentified emission line in the stacked X-ray spectrum of galaxy clusters, Astrophys. J. 789 (2014) 13 [arXiv:1402.2301] [INSPIRE]. 
[59] A. Boyarsky, O. Ruchayskiy, D. Iakubovskyi and J. Franse, An unidentified line in X-ray spectra of the andromeda galaxy and perseus galaxy cluster, arXiv:1402.4119 [INSPIRE].

[60] A.D. Dolgov, S.L. Dubovsky, G.I. Rubtsov and I.I. Tkachev, Constraints on millicharged particles from Planck data, Phys. Rev. D 88 (2013) 117701 [arXiv:1310.2376] [InSPIRE].

[61] P. Langacker and G. Steigman, Requiem for an FCHAMP? Fractionally CHArged, Massive Particle, Phys. Rev. D 84 (2011) 065040 [arXiv:1107.3131] [inSPIRE].

[62] Y. Nambu, Axial vector current conservation in weak interactions, Phys. Rev. Lett. 4 (1960) 380 [INSPIRE].

[63] Y. Nambu and G. Jona-Lasinio, Dynamical model of elementary particles based on an analogy with superconductivity. 1, Phys. Rev. 122 (1961) 345 [INSPIRE].

[64] Y. Nambu and G. Jona-Lasinio, Dynamical model of elementary particles based on an analogy with superconductivity. 2, Phys. Rev. 124 (1961) 246 [INSPIRE].

[65] S.P. Klevansky, The Nambu-Jona-Lasinio model of quantum chromodynamics, Rev. Mod. Phys. 64 (1992) 649 [INSPIRE].

[66] T. Hatsuda and T. Kunihiro, QCD phenomenology based on a chiral effective Lagrangian, Phys. Rept. 247 (1994) 221 [hep-ph/9401310] [INSPIRE].

[67] T. Kunihiro and T. Hatsuda, A selfconsistent mean field approach to the dynamical symmetry breaking: the effective potential of the Nambu-Jona-Lasinio model, Prog. Theor. Phys. 71 (1984) 1332 [INSPIRE].

[68] T. Hatsuda and T. Kunihiro, Fluctuation effects in hot quark matter: precursors of chiral transition at finite temperature, Phys. Rev. Lett. 55 (1985) 158 [INSPIRE].

[69] T. Kunihiro and T. Hatsuda, Effects of flavor mixing induced by axial anomaly on the quark condensates and meson spectra, Phys. Lett. B 206 (1988) 385 [Erratum ibid. 210 (1988) 278] [INSPIRE].

[70] J.H. Lowenstein and W. Zimmermann, Infrared convergence of Feynman integrals for the massless $A^{4}$ model, Commun. Math. Phys. 46 (1976) 105 [inSPIRE].

[71] J.H. Lowenstein and W. Zimmermann, The power counting theorem for Feynman integrals with massless propagators, Commun. Math. Phys. 44 (1975) 73 [Lect. Notes Phys. 558 (2000) 310] [INSPIRE].

[72] E.C. Poggio and H.R. Quinn, The infrared behavior of zero-mass Green's functions and the absence of quark confinement in perturbation theory, Phys. Rev. D 14 (1976) 578 [InSPIRE].

[73] Planck collaboration, P.A.R. Ade et al., Planck 2013 results. I. Overview of products and scientific results, arXiv: 1303.5062 [INSPIRE].

[74] XENON100 collaboration, E. Aprile et al., Dark matter results from 225 live days of XENON100 data, Phys. Rev. Lett. 109 (2012) 181301 [arXiv:1207.5988] [INSPIRE].

[75] LUX collaboration, D.S. Akerib et al., First results from the LUX dark matter experiment at the Sanford Underground Research Facility, Phys. Rev. Lett. 112 (2014) 091303 [arXiv:1310.8214] [INSPIRE].

[76] XENON1T collaboration, E. Aprile, The XENON1T dark matter search experiment, Springer Proc. Phys. 148 (2013) 93 [arXiv:1206.6288] [InSPIRE].

[77] J.M. Jauch and F. Rohrlich, The theory of photons and electrons, Addison-Wesley Pub. Company Inc., U.S.A. (1959). 
[78] H. Suganuma and T. Tatsumi, On the behavior of symmetry and phase transitions in a strong electromagnetic field, Annals Phys. 208 (1991) 470 [INSPIRE].

[79] S.P. Klevansky, J. Janicke and R.H. Lemmer, Collective modes of the Nambu-Jona-Lasinio model with an external U(1) gauge field, Phys. Rev. D 43 (1991) 3040 [INSPIRE].

[80] S. Tulin, H.-B. Yu and K.M. Zurek, Three exceptions for thermal dark matter with enhanced annihilation to $\gamma \gamma$, Phys. Rev. D 87 (2013) 036011 [arXiv: 1208.0009] [INSPIRE].

[81] S. Kanemura, S. Matsumoto, T. Nabeshima and H. Taniguchi, Testing Higgs portal dark matter via Z fusion at a linear collider, Phys. Lett. B 701 (2011) 591 [arXiv:1102.5147] [INSPIRE]. 